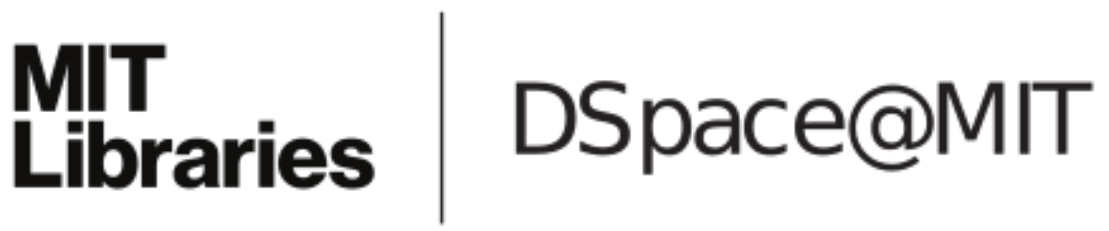

\author{
MIT Open Access Articles
}

Formation of beads-on-a-string structures

during break-up of viscoelastic filaments

The MIT Faculty has made this article openly available. Please share how this access benefits you. Your story matters.

Citation: Bhat, Pradeep P. et al. "Formation of Beads-on-a-string Structures During Break-up of Viscoelastic Filaments." Nat Phys 6.8 (2010) : 625-631.

As Published: $\mathrm{http}: / / \mathrm{dx}$.doi.org/10.1038/nphys1682

Publisher: Nature Publishing Group

Persistent URL: http://hdl.handle.net/1721.1/63090

Version: Author's final manuscript: final author's manuscript post peer review, without publisher's formatting or copy editing

Terms of use: Creative Commons Attribution-Noncommercial-Share Alike 3.0 


\title{
Formation of beads-on-a-string structures during breakup of viscoelastic filaments
}

\author{
Pradeep P. Bhat ${ }^{1}$, Santosh Appathurai ${ }^{1}$, Michael T. Harris ${ }^{1}$, Matteo \\ Pasquali $^{2,3}$, Gareth H. McKinley ${ }^{4}$ and Osman A. Basaran ${ }^{1 *}$ \\ ${ }^{1}$ School of Chemical Engineering, Purdue University, West Lafayette, IN 47907, USA \\ ${ }^{2}$ Department of Chemical and Biomolecular Engineering, Rice University, Houston, TX 77005, USA \\ ${ }^{3}$ Department of Chemistry, Rice University, Houston, TX 77005, USA \\ ${ }^{4}$ Department of Mechanical Engineering, Massachusetts Institute of Technology, Cambridge, MA 02139, USA \\ *e-mail: obasaran@purdue.edu
}

Breakup of viscoelastic filaments is pervasive in both nature and technology. If a filament is formed by placing a drop of saliva between a thumb and forefinger and is stretched, the filament's morphology close to breakup corresponds to beads of several sizes interconnected by slender threads. Although there is general agreement that formation of such beads-on-a-string (BOAS) structures only occurs for viscoelastic fluids, the underlying physics remains unclear and controversial. The physics leading to the formation of BOAS structures is probed by numerical simulation. Computations reveal that viscoelasticity alone does not give rise to a small, satellite bead between two much larger main beads but that inertia is required for its formation. Viscoelasticity, however, enhances the growth of the bead and delays pinch-off, which leads to a relatively long-lived beaded structure. We also show for the first time theoretically that yet smaller, sub-satellite beads can also form as seen in experiments.

Take a drop of saliva from the top of your tongue or between your cheek and gums, place it between your thumb and forefinger, and then pull your fingers slowly apart to a distance of about a centimeter. With a little practice, you will see a complex, poorly understood, and practically relevant non-Newtonian fluid dynamical process evolve before your eyes. The small thread of fluid saliva first starts to thin and drain under the action of capillarity but rather than rapidly breaking - as a thread of a Newtonian fluid like water would - it persists and evolves into a periodic pattern of beads strung together as a fluid necklace as shown in Figure 1(a). What forces are at play here? What determines the lifetime of the filament?

Upon closer observation, such phenomena can be found pervasively in nature, as in fish 
slimes, cellular protoplasm, and silk threads ${ }^{1}$. They are also encountered in important commercial processes such as anti-misting ${ }^{2}$ and electrospinning ${ }^{3}$. As D'Arcy Thompson ${ }^{4}$ noted in his observations of viscous plant protoplasm squirted out into a sugar solution of equal density, "the protoplasmic cylinder ... shows signs of instability and commences to disrupt; it tends to gather into spheres ... and in between these spheres we have more or less regularly alternate ones of smaller size."

In his seminal work, C. V. Boys ${ }^{1}$ demonstrated that a beads-on-a-string (BOAS) morphology can be reproduced by considering the capillary thinning of an annular film of a viscous Newtonian liquid on a central rigid core fiber, e.g., a piano wire. Subsequently, careful experiments and simulations of this process ${ }^{5-7}$ have revealed the essential physics: the initial wavelength of capillary instability in a cylindrical jet ${ }^{8}$ is modified by the solid core and the breakup of the fluid layer is suppressed by the viscous stress acting between the thinning fluid interface and the central fiber. This leads to a new annular film or 'collar' which thins further and subsequently becomes unstable once more, resulting in an iterated instability. By contrast, the drop of saliva shown in Figure 1(a) has no rigid cylindrical core; yet, in our digital rheometer, it displays a BOAS morphology. Therefore, the formation of the bead necklace must have a different origin in such fluids. One key requirement for the formation of beads in whole saliva is the presence of long chains of highly extensible polymer molecules such as mucopolysaccharides ${ }^{9}$ that impart viscoelasticity to the fluid. It is widely accepted that the large viscoelastic stresses resulting from the elongation of these macromolecules resist thinning and play the same role as the rigid core. Here we show that, though necessary, viscoelasticity alone is not sufficient for the formation of beaded structures. Moreover, we show that the BOAS phenomenon relies on the delicate interplay of four forces: capillary, viscous, elastic, and inertial. Indeed, when any of these forces dominates the others, it can overwhelm the dynamics of bead formation.

The material properties of saliva vary across the population but typical values of saliva's density $\rho$, zero-shear-rate viscosity $\eta_{0}$, and surface tension $\gamma\left(\rho \sim 1000 \mathrm{~kg} / \mathrm{m}^{3}, \eta_{0} \sim 1 \mathrm{mPa} \cdot \mathrm{s}\right.$, and $\gamma \sim 60 \mathrm{mN} / \mathrm{m})^{9,10}$ are not markedly different from those of water - a low-viscosity or nearly inviscid fluid. However, the lifetime of the thread of saliva (with an initial radius $R$ 
of, say, $1 \mathrm{~mm}$ ) is markedly longer than the simple estimate obtained by Rayleigh ${ }^{11}$ for an inviscid fluid $t_{c}=\sqrt{\rho R^{3} / \gamma} \sim 4 \mathrm{~ms}$. Viscous threads, for example the threads formed by a thumb and forefinger separating a thin film of pancake syrup (a viscous but essentially Newtonian fluid with constant viscosity $\eta_{0}$ ), live much longer with life times that scale with viscosity $t_{v}=\eta_{0} R / \gamma$. Viscous threads can also undergo iterated instabilities and form small, satellite droplets $^{12}$; however, they do not form a long-lived BOAS structure. Excessive elasticity also suppresses the formation of a BOAS structure ${ }^{13}$. This can be easily demonstrated with our own 'digital rheometer' as indicated in Figure 1(b). Moisten the lubricating strip at the leading edge of a razor cartridge assembly and let the water-soluble polymer film swell. When the swollen film is subsequently pressed onto a thumb or finger and withdrawn, multiple stable threads are formed. The long flexible polymer chains in the fluid elongate as the fluid columns thin under the action of capillarity. The resulting balance between elastic and capillary forces ${ }^{14}$ leads to a distinctive exponential rate of thinning in the filament diameter and provides a way of quantifying the characteristic relaxation time of the macromolecules ${ }^{15,16}$.

The importance of the different forces relative to one another in the dynamics of thinning and breakup of viscoelastic filaments is given primarily by two dimensionless parameters: the Ohnesorge number $\mathrm{Oh} \equiv t_{v} / t_{c}=\eta_{0} / \sqrt{\rho R \gamma}$, which represents the ratio of viscous to inertial forces (when the characteristic velocity scale is taken to equal $R / t_{c}$ ), and the Deborah number De $=\lambda / \tilde{t}$, which represents the ratio of the relaxation time $\lambda$ of the polymer molecules to the characteristic process time $\tilde{t}$. Depending on whether viscous or inertial force is dominant, the characteristic process time $\tilde{t}$ is either $t_{v}$ or $t_{c}$.

Numerous different configurations of fluid drops interconnected by thin filaments have been reported in studies of viscoelastic fluid pinch-off. Figure 1(c) depicts three of the most commonly occurring morphologies. The first consists of two primary or main drops connected by a thin and uniform fluid filament. This configuration has been studied by a number of authors ${ }^{16-19}$. The second consists of a satellite bead formed in between the main drops ${ }^{13,19-23}$ and the third consists of successive generations of smaller sub-satellite bead(s) formed in between the main drop and a satellite bead ${ }^{23-26}$. The first configuration 
is also seen in the pinch-off of very viscous $(\mathrm{Oh}>1)$ Newtonian filaments ${ }^{27}$ as shown by the computed interface shape of a Newtonian liquid bridge, i.e., a column of a Newtonian liquid constrained between two coaxial disks of equal radii, with $\mathrm{Oh}=2$ in Figure 1(d). However, unlike the viscoelastic case, the shapes of filaments connecting the beads in the Newtonian case are not axially uniform but show a smooth variation in the axial direction. The second configuration is also observed in the pinch-off of Newtonian fluids but only in the limit of low viscosity $(\mathrm{Oh}<1)^{28,29}$. This is evident from the shape of the pinching Newtonian filament with $\mathrm{Oh}=0.4$ in Figure 1(d). The third configuration, however, is observed only in the pinch-off of viscoelastic fluids, and computations to date have been unable to capture the formation of the sub-satellite beads.

Because there are many different potential drop-filament configurations, and because there are multiple forces at play, the physics of bead formation is still unclear, and the morphology in Figure 1(c) has been attributed to several different causes. For example, whereas Chang et al. ${ }^{21}$ predict that instability at the necks connecting the filaments and drops leads to successive generation of beads in an iterative fashion, Sattler et al. ${ }^{26}$ show that a sinusoidal instability which exists along the surface of the filament, together with successive contractions and relaxations of the interface at the ends of the filament can cause the formation of a beaded structure. Further, the recursive relation proposed by Chang et al. $^{21}$ for the radii of successive generation of filaments is inconsistent with experimental observation ${ }^{24}$ and their results showing the formation of a satellite bead when viscous force dominates the inertial force could not be reproduced in other simulations ${ }^{16}$. (For a detailed discussion, see pp. 69-70 of the review by Eggers and Villermaux ${ }^{30}$.) More importantly, the role of inertia in the dynamics has been either neglected or not clearly understood. Here, we elucidate the physics of bead formation during capillary-driven thinning of viscoelastic fluid filaments at finite De and Oh.

The spatio-temporal dynamics of fluid pinch-off is studied by computing the rupturing of an axisymmetric liquid bridge which is extended beyond its Rayleigh-Plateau stability limit $^{31}$. A schematic diagram of such a liquid bridge is shown in Figure 2. Because the problem is axisymmetric and gravity is not considered, only the domain highlighted in red is used 
in computations. The set of governing partial differential equations are solved using a robust finite element method ${ }^{32}$ that employs an elliptic mapping method ${ }^{33}$ to compute the deforming mesh. This method has been well benchmarked in diverse situations including analyses of coating flows ${ }^{34}$, Newtonian drop and filament breakup ${ }^{35,36}$, electrohydrodynamics ${ }^{37}$, and viscoelastic pinch-off ${ }^{38}$. (See Methods for the governing equations and the complete description of the problem.) Earlier experiments reporting BOAS formation in dilute solutions of polyethylene oxide $(\mathrm{PEO})$ in water ${ }^{23,39,40}$ yield the following range for the dimensionless parameters that we use in our simulations: $0.004 \leq \mathrm{De} \leq 0.5,0.01 \leq \mathrm{Oh} \leq 0.5$, and $\beta \geq 0.5$, where De is defined as De $=\lambda / t_{c}$ and $\beta=\eta_{s} / \eta_{0}$ is the viscosity ratio that represents the relative contribution of the solvent $\left(\eta_{s}\right)$ to the total viscosity $\eta_{0}=\eta_{s}+\eta_{p}$, where $\eta_{p}$ is the polymer viscosity.

Recently, Tirtaatmadja et al. ${ }^{13}$ have observed experimentally the formation of a single satellite bead during the dripping of drops of different PEO solutions which had similar viscosity and surface tension but different elastic properties. In particular, they observed that a satellite bead formed only at low Deborah number $(\mathrm{De}=0.0043,0.014$, and 0.031 ) but not at higher values ( $\mathrm{De}=0.1$ and 0.45$)$. A similar result is obtained here through computations as shown in Figure 3(a). A single satellite bead forms at De $=0.02$ but not at $\mathrm{De}=0.1$ and 0.5. Moreover, as De increases and elasticity becomes increasingly important, the filament initially thins faster and then slows down at later stages of pinch-off, in accord with an earlier nonlinear analysis ${ }^{20}$. All the fluids used by Tirtaatmadja et al. ${ }^{13}$ and in the other studies reporting the BOAS formation are low-viscosity fluids and, in particular, have low values of the Ohnesorge number $22-26,39$. Motivated by this observation, we next study the role of fluid inertia (i.e., $0<\mathrm{Oh}<1$ ) in the formation of beads.

It is well known that satellite drops also form during the pinch-off of low-viscosity Newtonian fluids ${ }^{29,41}$. Figure 3(b) shows the details of interface shapes of Newtonian and Oldroyd-B filaments near pinch-off (minimum neck radius $h_{\text {min }} / R \approx 1.18 \times 10^{-3}$ ) at the same Ohnesorge number as in Figure 3(a). Satellites form in both cases; however, in the viscoelastic case, the resulting satellite bead lives longer (because the thin interconnecting filament takes more time to break), becomes more spherical, and is roughly 1.6 times larger in volume than 
the Newtonian satellite. One may ask how does inertia cause the bead to form? A closer observation of the interface reveals that it evolves from an initially symmetric shape about the location of the minimum in the neck radius to an asymmetric shape. This is evident from the series of highly enlarged interface profiles shown at different times for a thinning Oldroyd-B filament in the lower part of Figure 3(b). The asymmetry that develops about the point of breakup (due to inertia ${ }^{28}$ ) leads to the creation of a satellite in both cases. However, the extended lifetime conferred by viscoelasticity enables the growth of the proto bead into a more spherical structure. As will be shown shortly, the additional stresses from the dissolved polymer molecules modify the flow and enhance the growth of the bead.

Christanti and Walker ${ }^{23}$, in their experiments with different PEO solutions, have observed that smaller, sub-satellite drops form when the wavelength of disturbance in jet breakup is long (they referred to these drops as sub-main drops; see Figure 4 of their paper). A similar behavior is also seen in the pinch-off of surfactant-laden Newtonian jets ${ }^{42}$. It is therefore natural to study the effect of domain size, specifically a higher aspect ratio $\Lambda=L / R$, on the formation of BOAS structures. Before we analyze viscoelastic breakup in the case of high aspect ratio threads, it proves informative to first investigate the corresponding effects for thinning Newtonian filaments. Thus, we consider the spatio-temporal dynamics of Newtonian filaments at an aspect ratio $\Lambda=3$ for two different values of Ohnesorge number; $\mathrm{Oh}=0.4$ (high inertia) and 0.7 (moderate inertia). Figure 4(a) makes it clear that close to pinch-off, the axial velocity profile in the Newtonian filament with larger inertia falls gradually near the center of the filament $(z / R=0)$ as opposed to that in the filament with lower inertia, where the fall is sharp. Indeed, at $\mathrm{Oh}=0.4$, the axial velocity $v_{z}$ in Figure 4(a) exhibits two maxima. Moreover, the axial component of the rate-of-strain tensor $D_{z z}=\partial v_{z} / \partial z$ forms a second local maximum close to $z / R \approx-0.27$, in addition to the one near the point of breakup. This can be seen in Figure 4(b), which also shows the interface shapes of the filaments in the same figure. A local maximum in the axial component of the rate-of-strain tensor corresponds to localized "pinching" and a local minimum in the neck radius. A second local maximum in $D_{z z}$ appears to form only at high inertia. This suggests that a thinner neck may develop locally in the region where $D_{z z}$ appears to form 
the second maximum. In the axial region between the two pinch points (where $D_{z z}$ is a local maximum at $\mathrm{Oh}=0.4$ ), there is a range of axial positions over which $D_{z z}$ is negative. Since the bridge shape over these values of $z$ is a slender thread, the flow is nearly one-dimensional and $v_{r}=-(r / 2) \partial v_{z} / \partial z$, where $v_{r}$ is the radial velocity ${ }^{28}$. Consequently, the radial velocity over this range of values of $z$ where $D_{z z}<0$ is positive and drives a local thickening of the neck. However, the filament pinches off at the first location before this thickening can develop. Thus, only one satellite forms in the Newtonian case.

Figure 4(c) shows that in a viscoelastic filament of Oh that is the same as the inertially dominated Newtonian filament $(\mathrm{Oh}=0.4)$, a small, sub-satellite bead forms between a main bead and a satellite bead. We next seek to understand what causes the formation of these secondary beads in the viscoelastic case. As in the case of the Newtonian filament at Oh = 0.4, once a viscoelastic filament has thinned sufficiently, there exists a range of values of $z$ for which $D_{z z}$ is negative, which indicates that the radial velocity is positive and the flow is not extensional. Further insights into the way viscoelasticity can impact the pinch-off dynamics beyond this stage can be gained by analyzing how the scalar quantity $\boldsymbol{\sigma}: \mathbf{D}$ (plotted in Figure 4(d)) varies along the filament length between the main drop and the satellite bead (see Methods). In regions where $\boldsymbol{\sigma}: \mathbf{D}$ is negative, an enhanced radial flow occurs. This leads to incipient growth of a small bead in the region $-0.49 \leq z / R \leq-0.46$ as evident from Figure 4(d). Unlike Newtonian filaments, pinch-off in viscoelastic filaments is delayed owing to high extensional stresses in the vicinity of the pinch point(s). Thus, this nascent bead has time to grow and a persistent BOAS structure ensues.

We next set out to map the range of De-Oh space into regions that exhibit, or do not exhibit, BOAS structures. To accomplish this, and to cover a wide range of De and Oh values expeditiously, we use a simplified one-dimensional analysis. (See Methods for details.) The aspect ratio is set as $\Lambda=3$ so that sub-satellites can also be seen in the dynamics, and the viscosity ratio is set as $\beta=0.6$. Figure 5 shows the corresponding phase diagram and indicates three distinct regions: those that show (1) no satellite bead formation, (2) a single central satellite bead, and (3) multiple generations of beads or a satellite and two sub-satellite beads. As is evident from the figure, inertia is necessary (i.e., Oh should be sufficiently small) 
for the formation of a bead, and sub-satellites form when both De and Oh are small. For a given $\mathrm{Oh}$, the morphology changes from a beaded structure to an axially uniform structure with increasing elasticity confirming the experimental observations of Tirtaatmadja et al. ${ }^{13}$ The product, $\mathrm{De} \cdot \mathrm{Oh}=\lambda t_{v} / t_{c}^{2}$, includes all three relevant time scales $\lambda$, $t_{v}$, and $t_{c}$ entering the problem, and it can be seen that the delineation of the region with no satellite beads from the one with a single satellite bead in Figure 5 is prescribed roughly by the curve De. $\mathrm{Oh}=0.2(\mathrm{Oh}+0.05)$ for $0.1 \leq \mathrm{Oh} \leq 1$ (curve not shown) - an observation that supports our argument that all relevant forces influence the detailed dynamics of bead formation. This local delineation of the two regions is not valid for higher Ohnesorge numbers $(\mathrm{Oh}>1)$ where the viscous force is dominant and the bead formation is suppressed.

The aforementioned results conclusively show that inertia is needed to induce the formation of a satellite bead. Indeed, when inertia is small, no bead formation is seen experimentally even at very high aspect ratios (see, e.g., Figure 1 of Clasen et al. ${ }^{16}$, where the authors show experimental images of the thinning of a high-viscosity, low surface tension polymeric thread at $\mathrm{Oh}=222)$. In simulations, however, there have been reports of bead formation in the absence of inertia ${ }^{43}$ but only when the fluid was modeled using the Phan-Thien/Tanner (PTT) network model incorporating non-affine motion. A recent study of this phenomenon establishes that the formation of a bead-like structure in these simulations is due to the unphysical behavior of the PTT model in strong transient flows which leads to spurious oscillations in the shear stress ${ }^{44}$.

While the analysis presented in this article answers the long-standing question of how BOAS morphologies form, a number of unanswered questions and avenues for further inquiries remain. First, the system studied here pertains to dilute polymer solutions with infinite chain extensibility. Incorporating finite extensibility (via the FENE dumbbell model, for example) ensures that the viscoelastic filament will always break eventually when the chains reach full extension ${ }^{21,38,45}$. This local pinch-off truncates the lifetime of the thinning filament and can interfere with the formation of multiple generations of beads. The corresponding phase diagram would then depend on an additional, independent dimensionless parameter (the chain extensibility). We have deliberately excluded such complications in the present 
study in order to focus exclusively on the key interactions of inertia, elasticity, and viscosity in controlling bead formation.

In a recent study involving more concentrated polymer solutions ${ }^{26}$, evaporation is observed to play a key role as a solidified beaded structure results at the end of the experiment. For these systems, additional physics, such as mass transfer at the liquid-gas interface due to drying, must be incorporated into the analysis. The occurrence of a solid beaded structure naturally leads one to wonder whether different types of structures can be obtained by incorporating noise into the simulations. Whereas the influence of noise in pinch-off dynamics has been considered in studies of Newtonian filaments ${ }^{46,47}$, no such studies have been carried out to date with viscoelastic filaments. Comparison of experimental observations with computational predictions of pinch-off dynamics of viscoelastic filaments and formation of BOAS morphologies can also provide stringent tests for validating existing constitutive equations and may offer a heretofore unexplored way of developing improved constitutive relations for materials deformed far from equilibrium.

\section{Methods}

Governing equations. The dynamics of pinch-off of fluids is governed by the conservation

of mass and momentum. For incompressible fluids, in the absence of gravity (neglected here as it is unimportant in most applications involving drop formation and in capillary thinning studies of thin fluid filaments), the conservation equations are

$$
\begin{aligned}
0 & =\boldsymbol{\nabla} \cdot \mathbf{v} \\
\rho\left(\frac{\partial \mathbf{v}}{\partial t}+\mathbf{v} \cdot \boldsymbol{\nabla} \mathbf{v}\right) & =\boldsymbol{\nabla} \cdot \mathbf{T}
\end{aligned}
$$

where $\mathbf{v}$ is the fluid velocity, $\mathbf{T}$ is the Cauchy stress tensor, and $\boldsymbol{\nabla}=\partial / \partial \mathbf{x}$ is the usual spatial gradient operator. The stress tensor $\mathbf{T}$ is split into an isotropic part, a viscous part, and an elastic part as $\mathbf{T}=-p \mathbf{I}+\boldsymbol{\tau}+\boldsymbol{\sigma}$, where $p$ is the pressure, $\mathbf{I}$ is the identity tensor, and the viscous stress tensor is $\boldsymbol{\tau}=2 \eta_{s} \mathbf{D}$, where $\mathbf{D}=(1 / 2)\left[\boldsymbol{\nabla} \mathbf{v}+(\boldsymbol{\nabla} \mathbf{v})^{T}\right]$ is the rate-of-strain tensor.

The elastic contribution to the Cauchy stress is obtained here using a conformation 
tensor based formalism ${ }^{48}$, and the Oldroyd-B constitutive equation is used to model the viscoelasticity of dilute polymer solution. For this model, the transport equation for the dimensionless conformation tensor $\mathbf{M}$ of the molecules is

$$
\frac{\partial \mathbf{M}}{\partial t}+\mathbf{v} \cdot \nabla \mathbf{M}-(\boldsymbol{\nabla} \mathbf{v})^{T} \cdot \mathbf{M}-\mathbf{M} \cdot \nabla \mathbf{v}=-\frac{1}{\lambda}(\mathbf{M}-\mathbf{I})
$$

and the relation between the elastic stress tensor and conformation tensor is $\boldsymbol{\sigma}=G(\mathbf{M}-\mathbf{I})$, where $G=\eta_{p} / \lambda$ is the elastic modulus.

Problem description. Figure 2 is a schematic diagram of an entire liquid bridge of volume $V$ and length $L$ held between two axisymmetric disks with equal radius $R$. The problem domain is highlighted in red color. Computations start with a quiescent fluid $(\mathbf{v}=\mathbf{0}$, $p=0$, and $\boldsymbol{\sigma}=\mathbf{0})$ that has an initially deformed interface $h / R=1-0.8 \cos (\pi z / L)$, where $-L / 2 \leq z \leq 0$. The dimensionless volume $\mathcal{V}=V / \pi R^{2} L$ of this liquid bridge is 0.30141, which, for the liquid bridge aspect ratios studied here, is unstable to axisymmetric perturbations ${ }^{31}$.

The no-slip and no-penetration boundary condition are applied along the solid disk. Along the axis of symmetry $S_{a}$ and the plane of symmetry $S_{p}$, no penetration and vanishing tangential stress conditions are applied. Finally, along the free surface, the traction boundary condition and the kinematic boundary condition are imposed.

The scalar quantity $\boldsymbol{\sigma}:$ D. A positive value of this quantity in a region indicates that polymer molecules in that region undergo axial elongation due to the local fluid motion, and a negative value of this quantity indicates that polymer molecules in that region undergo recoil, i.e., the conformation of the polymer chains evolves such that the molecules exert an axial traction on fluid elements and (via incompressibility) a radial thrust ${ }^{38}$.

One-dimensional analysis. The field variables (pressure, velocity, and the elastic stress) can be expanded in Taylor series in the radial coordinate $r$ and substituted in the governing equations and boundary conditions. Including only the terms of leading order in the resulting expressions, a set of one-dimensional (in space) partial differential equations are obtained 
(see Eggers and DuPont ${ }^{49}$ and Yarin ${ }^{18}$ for more details) which are solved here using the finite element method ${ }^{41,50}$. We have verified by comparing one- and two-dimensional (1D and 2D) computations that the 1D algorithm captures the details of the phase diagram with quantitative accuracy.

Pituity. The tendency to retard capillary breakup and extend the lifetime of low-viscosity polymeric filaments is sometimes referred to as 'pituity'.

\section{Acknowledgments}

This work was supported by the National Science Foundation Engineering Research Center for Structured Organic Particulate Systems (NSF ERC-SOPS)(EEC-0540855) at Rutgers, Purdue, NJIT, and UPRM.

\section{Author contributions}

P.P.B. planned research, performed calculations, analyzed data, and wrote the article; S.A. performed calculations and analyzed data; M.T.H. planned research; M.P. planned research, analyzed data, and wrote the article; G.H.M. performed experiments, analyzed data, and wrote the article; O.A.B. planned research, analyzed data, and wrote the article. 
(a)

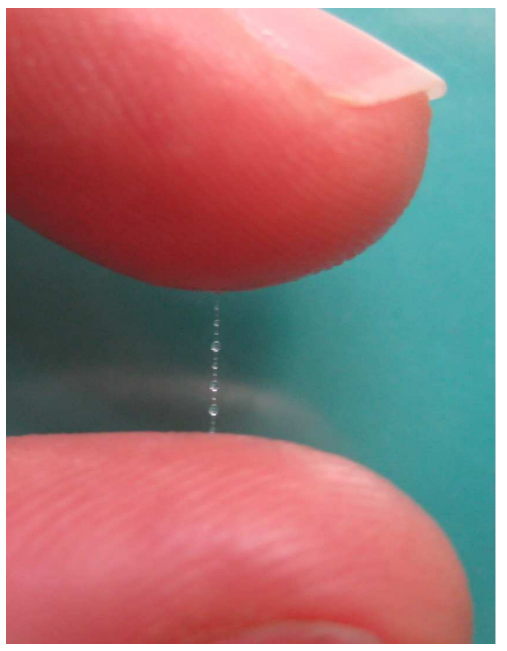

(c)

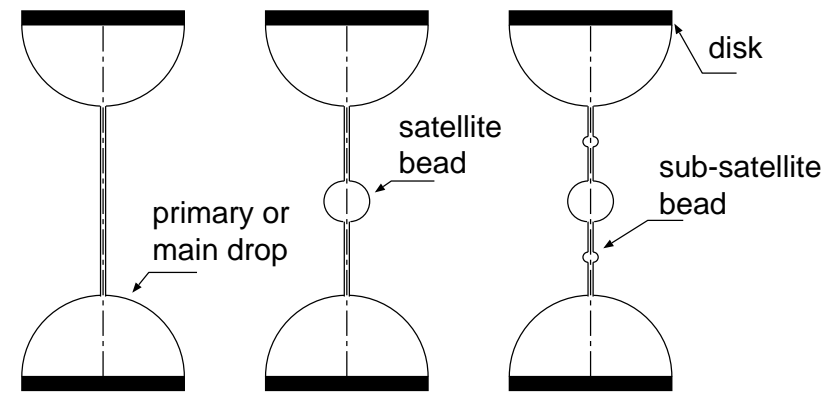

(b)

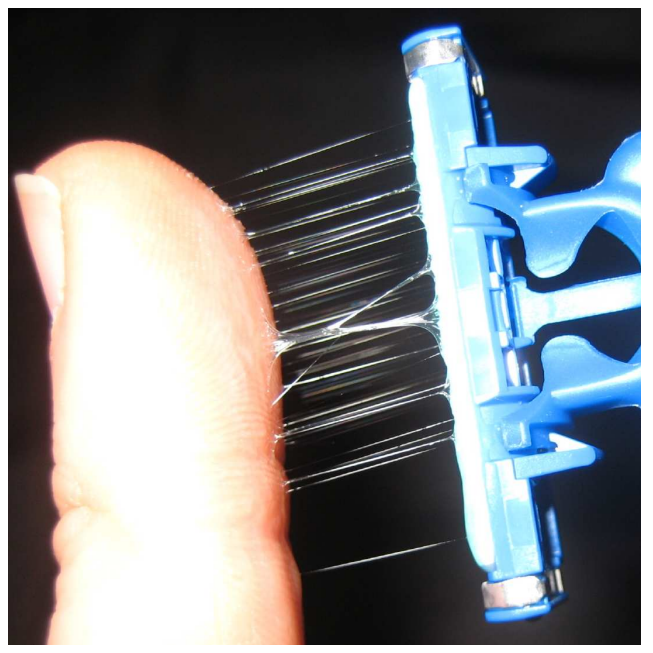

(d)

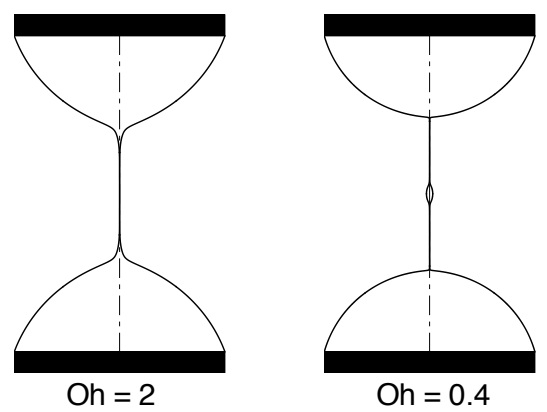

Figure 1: Using a 'digital rheometer' to measure the stringiness or pituity (see Methods) of various complex liquids, and filament morphologies near pinch-off. (a) a drop of sublingual saliva placed between thumb and forefinger and elongated to form a beads-on-a-string morphology and (b) a film of water soluble polymer elongated from the lubricating strip on a disposable razor. The enhanced viscosity and elasticity of the synthetic polymer solution in (b) results in a slippery and lubricious film but inhibits the formation of beads. (c) Sketches of viscoelastic filaments exhibiting different beads-on-a-string morphologies reported in the literature. (See text for details.) (d) Computed interface shapes of pinching Newtonian liquid bridges with aspect ratio $\Lambda=3$. Left: breakup of a high-viscosity filament $(\mathrm{Oh}=2)$, and right: breakup of a low-viscosity filament $(\mathrm{Oh}=0.4)$. The dash-dot line in $(\mathrm{c})$ and $(\mathrm{d})$ is the axis of symmetry. 


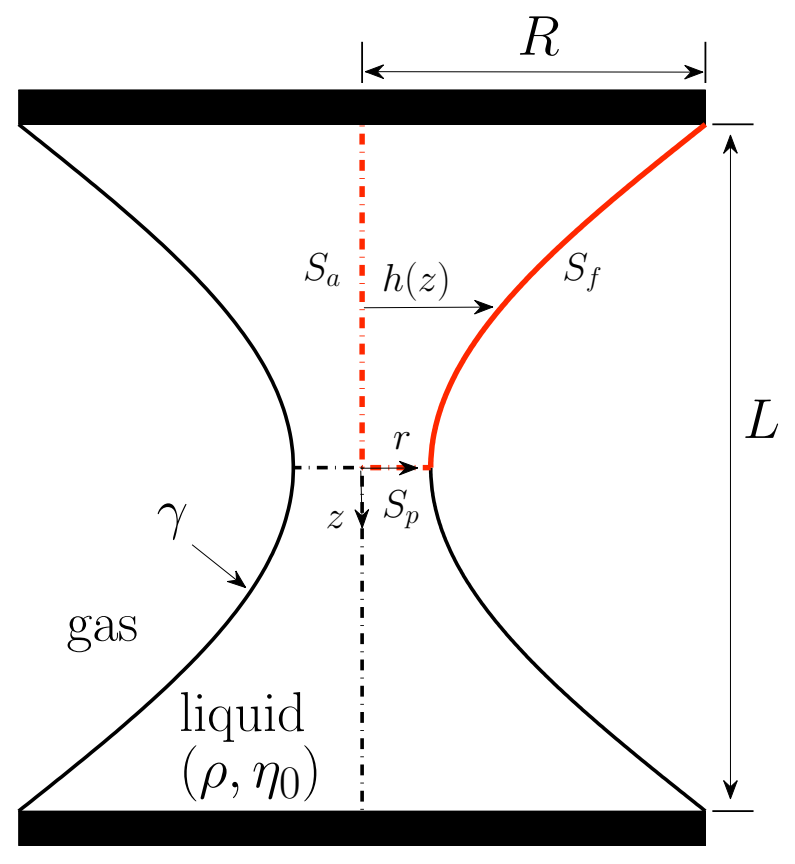

Figure 2: Liquid bridge definition sketch. $\rho$ is the density of the liquid, $\eta_{0}$ is the zero-shear-rate viscosity, and $\gamma$ is the surface tension. $L$ is the length of the bridge, $R$ is the radius of the disks, and $h(z)$ is the initial shape of the interface. Using symmetry, only the domain highlighted in red color is solved for in computations. $S_{f}, S_{a}$, and $S_{p}$ denote the liquid-gas interface, axis of symmetry, and plane of symmetry, respectively. 
(a)

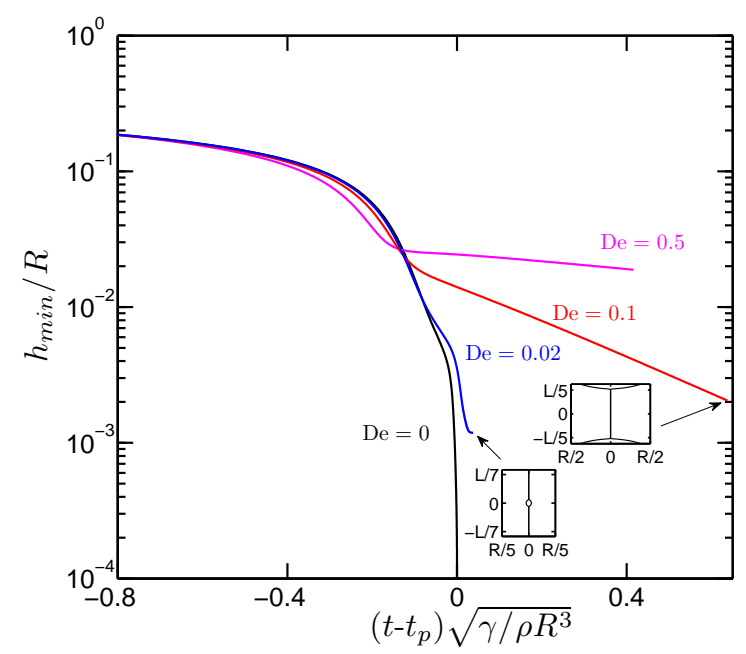

(b)
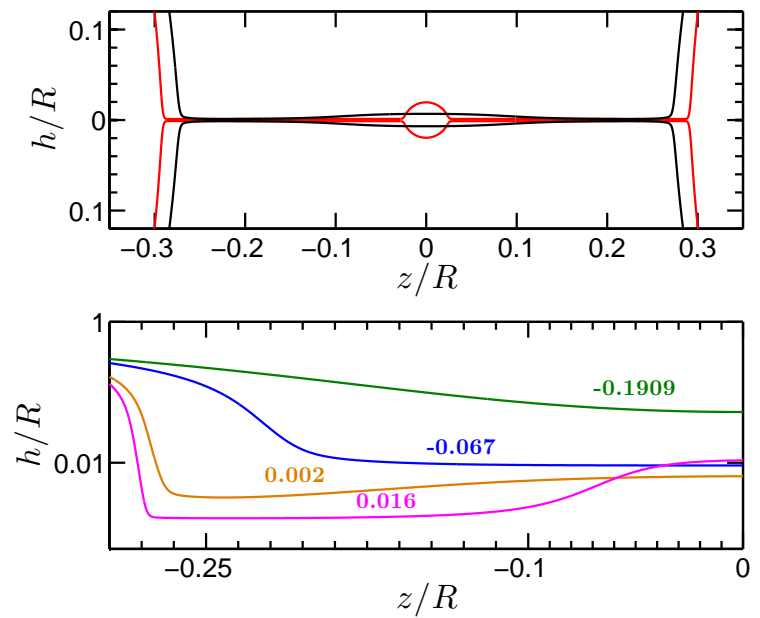

Figure 3: Filament thinning and satellite bead formation in Newtonian and Oldroyd-B filaments. (a) Variation of the minimum neck radius $h_{\min } / R$ with time in thinning Oldroyd-B filaments. The parameters are $\mathrm{Oh}=0.2, \beta=0.85$, and $\Lambda=1.5$ in all figures. The insets show the details of the interface at the very center of the liquid bridge. $t_{p}$ is the time at which $h_{\min } / R=10^{-4}$ in the Newtonian $(\mathrm{De}=0$ ) case. Formation of a satellite bead is seen when the Deborah number is small (here, $\mathrm{De}=0.02$ ) but not when it is high $(\mathrm{De}=0.1$ and 0.5$)$. (b) Top: interface shapes of Oldroyd-B (red line) and Newtonian (black line) filaments near pinch-off. In the viscoelastic fluid $\mathrm{De}=0.02$, and $h_{\min } / R \approx 1.18 \times 10^{-3}$ in both cases. Satellites form in both filaments, but in the viscoelastic case, the satellite bead formed is more spherical and has a volume which is roughly 1.6 times greater than that in the Newtonian case. Bottom: highly enlarged views (around the pinch point) of the interface shapes of the Oldroyd-B filament at different dimensionless times $\left(t-t_{p}\right) / t_{c}$ before (negative) and after (positive) the time of pinch-off $t_{p}$ for the Newtonian fluid. 
(a)

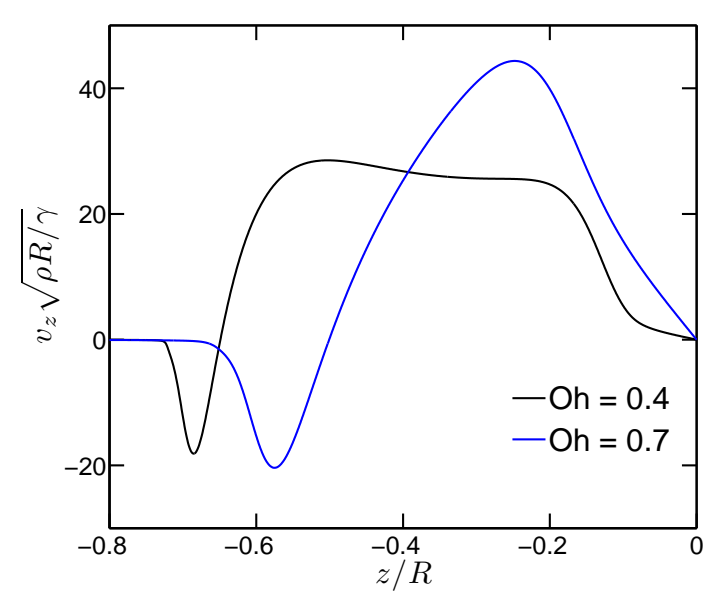

(c)

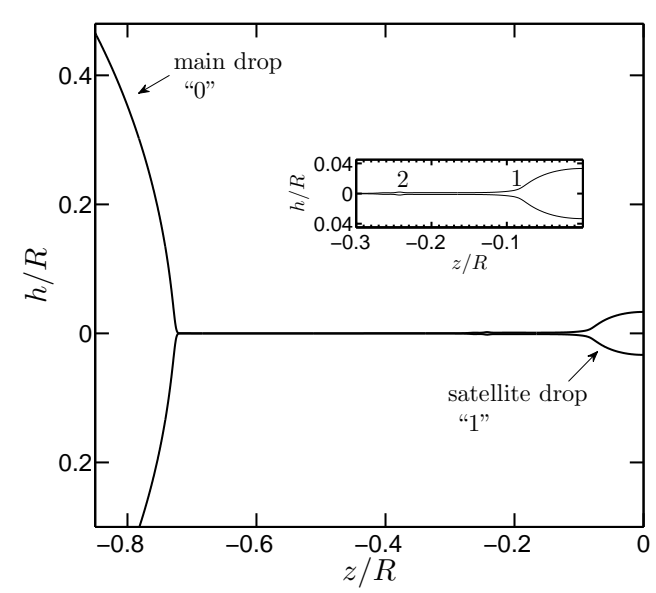

(b)

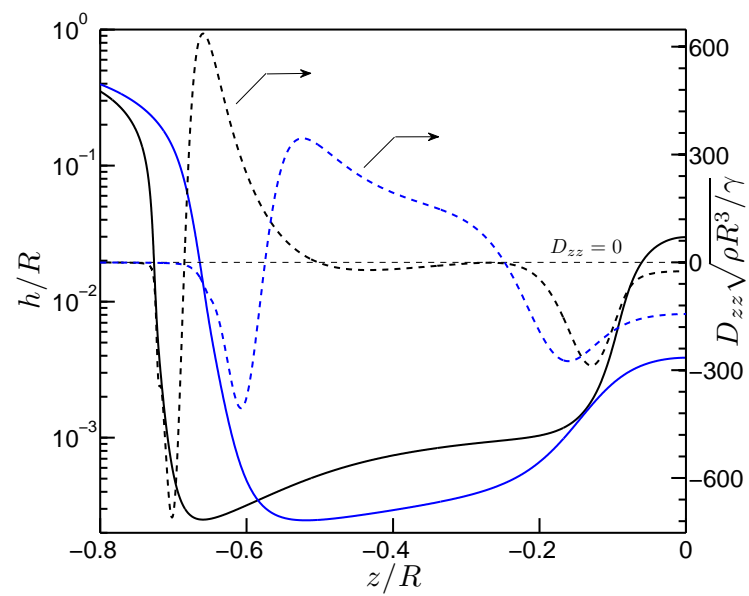

(d)

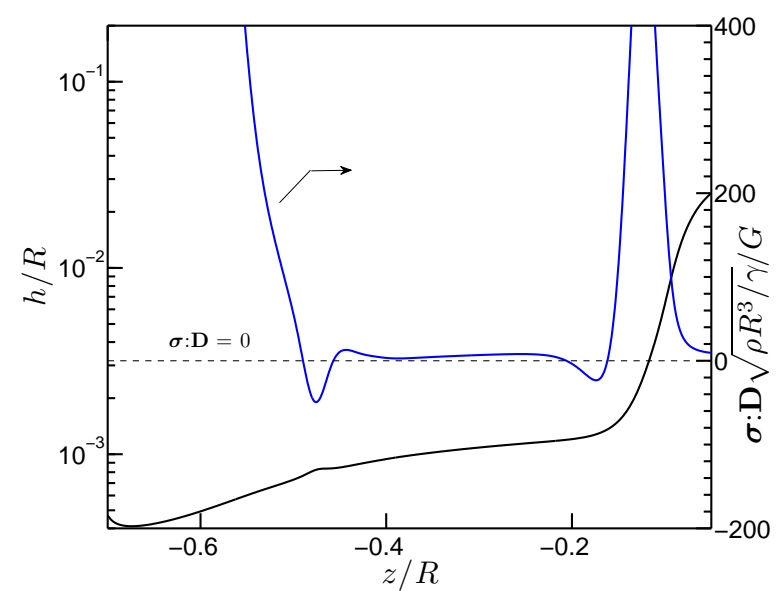

Figure 4: Influence of fluid inertia on the dynamics of satellite bead formation, and formation of sub-satellite beads in viscoelastic filaments. (a) Variation of the axial velocity along the interface in Newtonian filaments at $\mathrm{Oh}=0.7$ (blue line) and $\mathrm{Oh}=0.4$ (black line). The minimum neck radius $h_{\min } / R \approx 2.5 \times 10^{-4}$ in the two cases. The aspect ratio $\Lambda$ is 3 in all figures. (b) Interface shapes (solid lines) and the axial component of the rate-of-strain tensor $D_{z z}$ (dashed lines) along the free surface in Newtonian filaments at the same values of the Ohnesorge number as studied in (a). (c) Formation of a secondary satellite bead in an Oldroyd-B filament. Here, $h_{\text {min }} / R=2.2 \times 10^{-4}$. The inset shows the magnified view of the region containing the satellite and the sub-satellite bead. The parameters are $\mathrm{Oh}=0.4, \mathrm{De}=4 \times 10^{-3}$, and $\beta=0.5$. (d) Variation of $\boldsymbol{\sigma}: \mathbf{D}$ (blue line) along the free surface and the interface shape profile (black line) in an Oldroyd-B filament. The parameters are the same as in (c). 

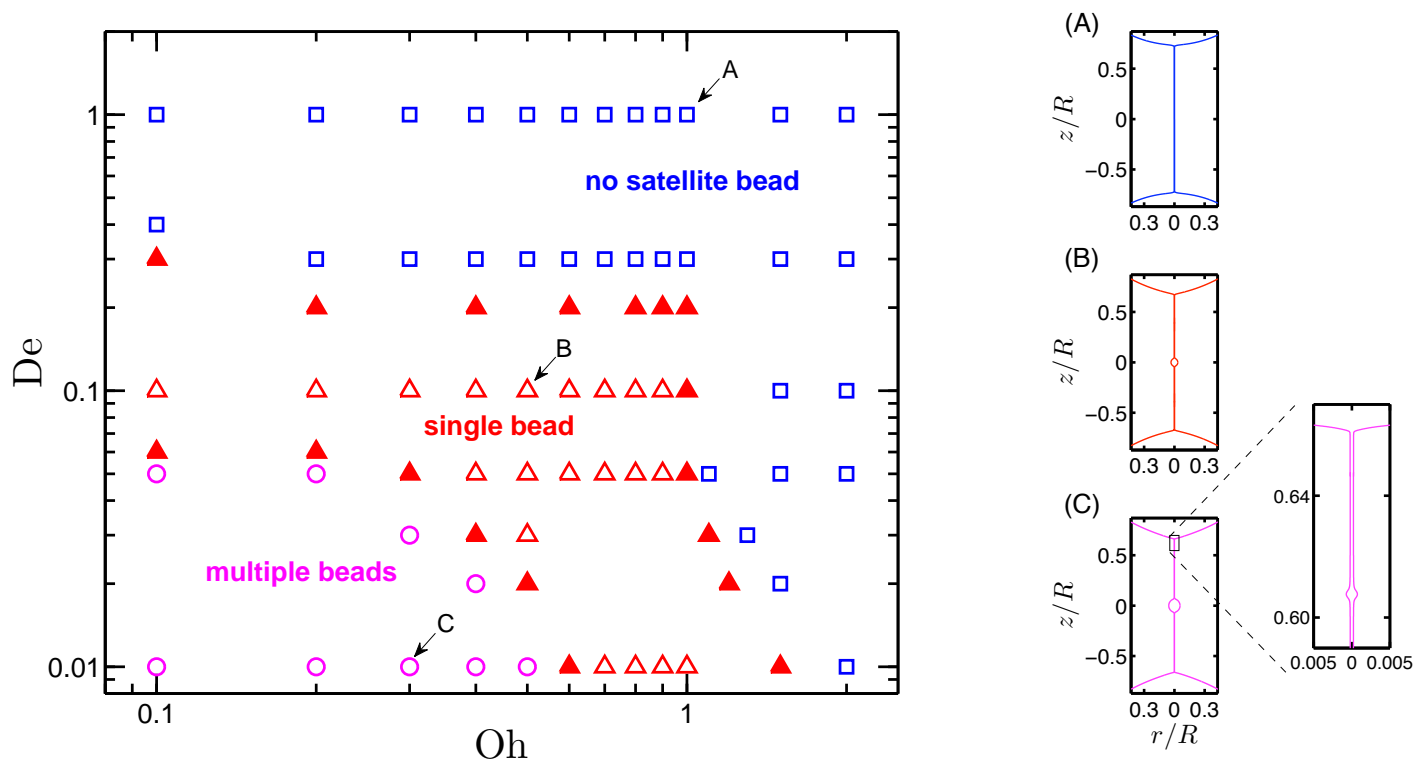

Figure 5: Phase diagram depicting the regions showing different beads-on-a-string morphologies in the De and Oh space for Oldroyd-B filaments with $\beta=0.6$ and $\Lambda=3$. The blue squares represent uniform cylindrical threads (i.e., no satellite beads); the red triangles indicate the occurrence of a single satellite bead; and the magenta circles indicate that sub-satellite beads develop between the main drop attached to the end plates and the primary satellite bead that is at the center of the filament. The filled triangles represent the first occurrence of the single satellite bead morphology when transitioning from either of the other two regions. Representative filament shapes corresponding to each of the three morphologies (points A, B, and C in the phase diagram) are shown on the right. 


\section{References}

1. Boys, C. V. Soap bubbles and the forces which mould them (Doubleday Anchor Books, Garden City, N. Y., 1959).

2. Hoyt, J. W. \& Taylor, J. J. Turbulence structure in a water jet discharging in air. Phys. Fluids 20, S253-S257 (1977).

3. Reneker, D. H., Yarin, A. L., Zussman, E. \& Xu, H. Electrospinning of nanofibres from polymer solutions and melts. Adv. Appl. Mech. 41, 43-195 (2007).

4. Thompson, D. W. On growth and form (Cambridge University Press, Cambridge, 1917).

5. Goren, S. L. The instability of an annular thread of fluid. J. Fluid Mech. 12, 309-319 (1962).

6. Goren, S. L. The shape of a thread of liquid undergoing break-up. J. Colloid Sci. 19, 81-86 (1964).

7. Kalliadasis, S. \& Chang, H.-C. Drop formation during coating of vertical fibres. J. Fluid Mech. 261, 135-168 (1994).

8. Eggers, J. Nonlinear dynamics and breakup of free-surface flows. Rev. Modern Phys. 69, 865-929 (1997).

9. Schipper, R. G., Silletti, E. \& Vingerhoeds, M. H. Saliva as research material: Biochemical, physicochemical and practical aspects. Arch. Oral Biol. 52, 1114-1135 (2007).

10. Stokes, J. R. \& Davies, G. A. Viscoelasticity of human whole saliva collected after acid and mechanical stimulation. Biorheology 44, 141-160 (2007).

11. Rayleigh, L. On the instability of jets. Proc. Lond. Math. Soc. 10, 4-13 (1878).

12. Shi, X. D., Brenner, M. P. \& Nagel, S. R. A cascade of structure in a drop falling from a faucet. Science 265, 219-222 (1994).

13. Tirtaatmadja, V., McKinley, G. H. \& Cooper-White, J. J. Drop formation and breakup of low viscosity elastic fluids: Effects of molecular weight and concentration. Phys. Fluids. 18, 043101 (2006).

14. Entov, V. M. \& Hinch, E. J. Effect of a spectrum of relaxation times on the capillary thinning of a filament of elastic liquid. J. Non-Newtonian Fluid Mech. 72, 31-53 (1997).

15. Bazilevskii, A. V., Entov, V. M. \& Rozhkov, A. N. Breakup of an Oldroyd liquid bridge as a method for testing the rheological properties of polymer solutions. Polym. Sci. Ser. A 43, 716-726 (2001).

16. Clasen, C., Eggers, J., Fontelos, M. A., Li, J. \& McKinley, G. H. The beads-on-string structure of viscoelastic threads. J. Fluid Mech. 556, 283-308 (2006). 
17. Goldin, M., Yerushalmi, H., Pfeffer, R. \& Shinnar, R. Breakup of a laminar capillary jet of a viscoelastic fluid. J. Fluid Mech. 38, 689-711 (1969).

18. Yarin, A. L. Free liquid jets and films: hydrodynamics and rheology (Longman Scientific \& Technical, New York, 1993).

19. Li, J. \& Fontelos, M. A. Drop dynamics on the beads-on-string structure for viscoelastic jets: A numerical study. Phys. Fluids 15, 922-937 (2003).

20. Bousfield, D. W., Keunings, R., Marrucci, G. \& Denn, M. M. Nonlinear analysis of the surface tension driven breakup of viscoelastic filaments. J. Non-Newtonian Fluid Mech. 21, 79-97 (1986).

21. Chang, H.-C., Demekhin, E. A. \& Kalaidin, E. Iterated stretching of viscoelastic jets. Phys. Fluids 11, 1717-1737 (1999).

22. Wagner, C., Amarouchene, Y., Bonn, D. \& Eggers, J. Droplet detachment and satellite bead formation in viscoelastic fluids. Phys. Rev. Lett. 95, 164504 (2005).

23. Christanti, Y. \& Walker, L. M. Surface tension driven jet breakup of strain-hardening polymer solutions. J. Non-Newtonian Fluid Mech. 100, 9-26 (2001).

24. Oliveira, M. S. N. \& McKinley, G. H. Iterated stretching and multiple breads-on-a-string phenomena in dilute solutions of highly extensible flexible polymers. Phys. Fluids 17, 071704 (2005).

25. Oliveira, M. S. N., Yeh, R. \& McKinley, G. H. Iterated stretching, extensional rheology and formation of beads-on-a-string structures in polymer solutions. J. Non-Newtonian Fluid Mech. 137, 137-148 (2006).

26. Sattler, R., Wagner, C. \& Eggers, J. Blistering pattern and formation of nanofibers in capillary thinning of polymer solutions. Phys. Rev. Lett. 100, 164502 (2008).

27. Papageorgiou, D. T. On the breakup of viscous liquid threads. Phys. Fluids 7, 1529-1544 (1995).

28. Eggers, J. Universal pinching of 3D axisymmetric free surface flow. Phys. Rev. Lett. 71, 3458 (1993).

29. Notz, P. K., Chen, A. U. \& Basaran, O. A. Satellite drops: Unexpected dynamics and change of scaling during pinch-off. Phys. Fluids 13, 549-552 (2001).

30. Eggers, J. \& Villermaux, E. Physics of liquid jets. Rep. Prog. Phys. 71, 036601 (2008).

31. Slobozhanin, L. A. \& Perales, J. M. Stability of liquid bridges between equal disks in an axial gravity field. Phys. Fluids A 5, 1305-1314 (1993).

32. Pasquali, M. \& Scriven, L. E. Free surface flows of polymer solutions with models based on conformation tensor. J. Non-Newtonian Fluid Mech. 108, 363-409 (2002). 
33. de Santos, J. M. Two-phase Cocurrent Downflow through Constricted Passages. Ph.D. thesis, University of Minnesota, Minneapolis, MN (1991).

34. Christodoulou, K. N. \& Scriven, L. E. The fluid-mechanics of slide coating. J. Fluid Mech. 208, 321-354 (1989).

35. Chen, A. U., Notz, P. K. \& Basaran, O. A. Computational and experimental analysis of pinch-off and scaling. Phys. Rev. Lett. 88, 174501 (2002).

36. Suryo, R. \& Basaran, O. A. Tip streaming from a liquid drop forming from a tube in a co-flowing outer fluid. Phys. Fluids 18, 082102 (2006).

37. Collins, R. T., Jones, J. J., Harris, M. T. \& Basaran, O. A. Electrohydrodynamic tip streaming and emission of charged drops from liquid cones. Nature Phys. 4, 149-154 (2008).

38. Bhat, P. P., Basaran, O. A. \& Pasquali, M. Dynamics of viscoelastic filaments: Low capillary number flows. J. Non-Newtonian Fluid Mech. 150, 211-225 (2008).

39. Mun, R. P., Byars, J. A. \& Boger, D. V. The effects of polymer concentration and molecular weight on the breakup of laminar capillary jets. J. Non-Newtonian Fluid Mech. 74, 285-297 (1998).

40. Cooper-White, J. J., Fagan, J. E., Tirtaatmadja, V., Lester, D. R. \& Boger, D. V. Drop formation dynamics of constant low-viscosity, elastic fluids. J. Non-Newtonian Fluid Mech. 106, 29-59 (2002).

41. Ambravaneswaran, B., Wilkes, E. D. \& Basaran, O. A. Drop formation from a capillary tube: Comparison of one-dimensional and two-dimensional analyses and occurrence of satellite drops. Phys. Fluids 14, 2606-2621 (2002).

42. McGough, P. T. \& Basaran, O. A. Repeated formation of fluid threads in breakup of a surfactant-covered jet. Phys. Rev. Lett. 96, 054502 (2006).

43. Matallah, H., Banaai, M. J., Sujatha, K. S. \& Webster, M. F. Modelling filament stretching flows with strain-hardening models and sub-cell approximations. J. NonNewtonian Fluid Mech. 134, 77-104 (2006).

44. Bhat, P. P., Pasquali, M. \& Basaran, O. A. Beads-on-string formation during filament pinch-off: Dynamics with the PTT model for non-affine motion. J. Non-Newtonian Fluid Mech. 159, 64-71 (2009).

45. Clasen, C., Bico, J., Entov, V. \& McKinley, G. H. 'Gobbling drops': the jetting-dripping transition in flows of polymer solutions. J. Fluid Mech. 636, 5-40 (2009).

46. Moseler, M. \& Landman, U. Formation, stability, and breakup of nanojets. Science 289, 1165-1169 (2000). 
47. Eggers, J. Dynamics of liquid nanojets. Phys. Rev. Lett. 89, 084502 (2002).

48. Pasquali, M. \& Scriven, L. E. Theoretical modeling of microstructured liquids: a simple thermodynamic approach. J. Non-Newtonian Fluid Mech. 120, 101-135 (2004).

49. Eggers, J. \& DuPont, T. F. Drop formation in a one-dimensional approximation of the Navier-Stokes equation. J. Fluid Mech. 262, 205-221 (1994).

50. Zhang, X., Padgett, R. S. \& Basaran, O. A. Nonlinear deformation and breakup of stretching liquid bridges. J. Fluid Mech. 329, 207-245 (1996). 